\title{
Lidil
}

Revue de linguistique et de didactique des langues

$56 \mid 2017$

Grammaires et littéracies

\section{L'apprenant en FLE confronté à la polyphonie textuelle : littéracie, discours, grammaire}

French As a Foreign Language Learner Facing Text Polyphony: Literacy, Discourse, Grammar

Marie-Odile Hidden et Henri Portine

\section{OpenEdition}

\section{Journals}

Édition électronique

URL : http://journals.openedition.org/lidil/4766

DOI : $10.4000 /$ lidil.4766

ISSN : 1960-6052

Éditeur

UGA Éditions/Université Grenoble Alpes

Édition imprimée

ISBN : 978-2-37747-024-2

ISSN : 1146-6480

Référence électronique

Marie-Odile Hidden et Henri Portine, «L'apprenant en FLE confronté à la polyphonie textuelle:

littéracie, discours, grammaire », Lidil [En ligne], 56 | 2017, mis en ligne le 01 novembre 2017, consulté le 01 mai 2019. URL : http://journals.openedition.org/lidil/4766 ; DOI : 10.4000/lidil.4766

Ce document a été généré automatiquement le 1 mai 2019.

(C) Lidil 


\section{L'apprenant en FLE confronté à la polyphonie textuelle : littéracie, discours, grammaire}

French As a Foreign Language Learner Facing Text Polyphony: Literacy, Discourse, Grammar

Marie-Odile Hidden et Henri Portine

\section{Introduction}

1 Selon le Cadre européen commun de référence pour les langues ${ }^{1}$ (2001, p. 58), l'apprenant de niveau avancé ( $\mathrm{C} 1$ ) peut comprendre une gamme étendue de textes et «identifier des points de détail fins, y compris les attitudes, que les opinions soient exposées ou implicites ». Or, cette interprétation des différentes positions convoquées dans un texte à dominante argumentative ne va pas de soi, comme nous l'avons constaté lors d'une recherche menée avec des apprenants allophones de niveau B2/C1: les apprenants ont en effet tendance à considérer le texte comme le pur produit du scripteur qui ne délivre que sa parole (le discours rapporté étant alors simple discours de témoignage), et non comme la résultante d'un scripteur qui «fait parler» des protagonistes («polyphonie textuelle $\left.{ }^{2} »\right)$ afin d'orienter le lecteur vers sa propre position. Cela nous a amenés à étudier la façon dont ces apprenants gèrent la complémentarité des marques grammaticales à l'œuvre dans la polyphonie (notamment le conditionnel et le discours rapporté) et des niveaux textuel et intertextuel. Or cette intertextualité est aussi présente dans la notion de littéracie puisque celle-ci englobe la circulation des écrits (cf. cidessous). Nous traiterons donc du rapport entre la grammaire et la littéracie, cette dernière permettant de mettre en regard différents textes ou différentes positions sur un thème donné.

2 Dans un premier temps, nous dresserons le panorama de l'évolution du rôle de la grammaire en didactique d'une langue, de la grammaire pour structurer ses énoncés à la 
grammaire pour le discours, puis à la grammaire pour la littéracie en travaillant sur la prise en compte de la polyphonie textuelle. Nous décrirons ensuite deux marquages de cette polyphonie en français : le conditionnel et le discours rapporté, avant de présenter un travail empirique en classe de FLE à partir de textes de presse mettant en scène cette polyphonie. Enfin, à la lumière des réactions et des difficultés des apprenants, nous ferons des propositions didactiques concernant le contenu des grammaires pédagogiques et l'approche des textes.

\section{De la grammaire à la littéracie : aboutissement d'un parcours}

3 Établir un pont entre grammaire et littéracie n'est pas de l'ordre de l'évidence du fait même de l'histoire de la grammaire. Cela nécessite donc une contextualisation de notre problématique.

\subsection{De la grammaire pour structurer sa phrase à la grammaire pour le discours}

4 La grammaire est née dans le système éducatif à Alexandrie au II siècle av. J.-C. et donc dans un contexte pédagogique. Elle fut d'emblée située par rapport à l'apprentissage de la rhétorique (donc au service du discours, cf. Portine, 1999), malgré des applications souvent très normatives.

$5 \mathrm{Au} \mathrm{XIV}^{\mathrm{e}}$ siècle, son émancipation de la rhétorique fit de la grammaire une discipline autonome, notamment chez les Daces (grammairiens danois). Ce changement de paradigme a eu une conséquence: l'enseignement de la grammaire non plus comme auxiliaire de la production discursive mais pour elle-même en tant que science et apprentissage des structures de phrases. Un autre bouleversement a eu lieu à l'articulation des $\mathrm{XVIII}^{\mathrm{e}}$ et $\mathrm{XIX}^{\mathrm{e}}$ siècles : la constitution d'une grammaire scolaire (langue maternelle) orientée prioritairement vers l'acquisition de l'orthographe (Chervel, 1977), comme savoir autonome (notamment pour gérer les accords).

À partir de 1960, en français langue étrangère (ci-après, FLE), la méthodologie structuroglobale audio-visuelle (SGAV), qui donnait la priorité à l'oral, fit de la grammaire le lieu d'un apprentissage intuitif des structures phrastiques sous la forme d'exercices structuraux.

Dans le SGAV, le niveau phrastique jouait un rôle important et le niveau discursif n'était pris en compte qu'en fin de leçon. Cependant, le SGAV céda, en 1978, la place aux approches communicatives constituées d'abord dans le cadre de l'EFL (English as a Foreign Language) dès 1973. Parallèlement, des approches discursives (Sinclair \& Coulthard, 1975 pour l'anglais ; Lehmann et coll., 1979 et Lehmann, 1980 pour le français) commençaient à se développer. Mais ces travaux ne posaient pas le problème du rapport à la grammaire (traditionnellement peu audible pour l'anglais). Une exception fut la publication en français langue maternelle de deux manuels de $4^{\mathrm{e}}$ et de $3^{\mathrm{e}}$ qui cherchaient à relier grammaire et textes (Combettes, Fresson \& Tomassone, 1979-1980), la grammaire publiée par l'une des auteurs (Tomassone, 1996) partant du texte pour aller aux syntagmes et aux classes de mots. 
8 En langue étrangère, la question de la grammaire a hésité entre prise en compte ou non du discours (Beacco, 1989), de l'apprenant et de ses erreurs (Besse \& Porquier, 1984), des activités métalinguistiques (Bourguignon \& Foerster, 1993 ; Tisset, 1997). Cela nous amène à traiter, avant le rapport grammaire-discours, du rapport grammaire-apprenant qui émerge à partir du début des années 1980.

\subsection{La grammaire, composante d'un apprentissage linguistique}

ll'on considère que les exercices structuraux forment la façon d'exploiter la grammaire en didactique des langues, il y a « enseignement de la grammaire ». Mais si l'on se pose la question de l'implication de la grammaire dans l'activité discursive, on passe à une "didactique de la grammaire ». La différence est d'importance parce qu'elle recouvre deux démarches différentes: partir de la grammaire pour aller vers les textes ou le discours; partir des textes et du discours pour aller vers des phases de stabilisation grammaticale. Tel est l'enjeu de ce qui suit ${ }^{3}$. Afin de bien comprendre la différence, il convient de rappeler l'opposition entre opérations dites « de bas niveau » et opérations dites «de haut niveau» (nous avons choisi cette «théorisation» parce qu'elle est facilement opératoire du point de vue didactique) :

- les opérations de bas niveau travaillent sur les mots et syntagmes, de façon analytique (relier une terminaison verbale à un paradigme flexionnel; relier une construction à un modèle) ;

- les opérations de haut niveau prennent en considération la situation de production, les connaissances encyclopédiques (réseaux de concepts, normes socio-situationnelles, règles générales) pour élucider le sens d'un ensemble (paragraphe, chapitre, texte) et sont donc soit synthétiques soit holistiques.

Dans la compréhension et dans la production, le premier mouvement va des opérations de haut niveau à celles de bas niveau (mouvement dit top-down), puis ces opérations agissent dialectiquement, en interaction (cf. Portine, 1998). La grammaire permet à l'apprenant de régulariser les opérations de bas niveau au service du haut niveau (le discours).

11 Si l'on enseigne la grammaire, alors on fournit aux apprenants des formes et des règles ; à charge pour eux de les relier aux activités discursives. Si l'on cherche à construire une didactique de la grammaire, alors on part des textes et l'on cherche à faire relier, par les apprenants, des passages de ces textes à des points grammaticaux répertoriés comme tels mais aussi conçus comme des "moteurs de stratégies discursives ${ }^{4}$ ", sans oublier pour autant la grammaire comme "facteur de normes sociales» et comme "régulateur de structures » (Portine, 1999, p. 200-203). Cette conception dynamique ne fait pas produire automatiquement " du discours pertinent ", mais elle en est la condition première. C'est aussi ce type de prise en compte de la grammaire qui nous a conduits à élargir notre champ à la question de la littéracie parce que, si la didactique de la grammaire appliquée au discours fonctionne bien pour un texte homogène (qui fait peu de place à la polyphonie), ce n'est plus le cas pour des textes qui incluent une part importante de polyphonie, comme nous le verrons plus loin. 


\subsection{De la grammaire pour le discours à la grammaire pour la littéracie}

Goody et Watt (1962) ont montré comment l'écrit (literacy) avait profondément modifié les structures sociales, positivement (réflexion possible, encouragement de la production personnelle, partage du savoir dans le cas des écrits alphabétiques) et négativement (caractère abstrait, risque d'une croyance dénuée de fondements).

Le terme literacy ou littéracie (on trouve aussi littératie) recouvre divers usages :

- pour l'UNESCO, «une communauté possédant l'écrit (literate community) est une communauté dynamique ${ }^{5} »$, ce qui conduit à entendre la literacy comme allant de l'alphabétisation à la circulation de textes (correspondance administrative, échanges épistolaires - électroniques ou non - familiaux et amicaux, fairepart) ;

- pour l'OCDE (2000: page x de la préface), la littératie est l'«aptitude à comprendre et à utiliser l'information écrite dans la vie courante, à la maison, au travail et dans la collectivité en vue d'atteindre des buts personnels et d'étendre ses connaissances et ses capacités ", conception plus large, centrée sur la communication écrite ;

- pour J.-L. Chiss (2003, p. 158), au sens large, « le champ de la littératie intègre le savoirécrire et l'usage du langage écrit dans la société, ce qu'on pourrait nommer la culture de l'écrit. [...] l'étude de la littératie consiste à ausculter les modalités d'appropriation de l'écrit, les fonctions assignées à son emploi dans des systèmes sociaux différents où les partages entre oralité et écriture ne s'opèrent pas de la même manière ».

14 Nous nous réfèrerons ici à la définition de l'OCDE parce que celle de Chiss supposerait que l'on travaille sur la mise en réseau textuel à la fois en français et dans les langues de scolarisation des apprenants. Dès lors, du point de vue didactique, la littéracie englobera la construction d'un texte en production et sa reconstruction en compréhension ainsi que la prise en compte de la circulation des textes, ce qui permet de rendre compte de la polyphonie textuelle, c'est-à-dire du fait que les textes contiennent des traces d'autres textes (cf. ci-dessous). En nous appuyant sur les difficultés rencontrées par les apprenants lors de leurs apprentissages, nous avons sélectionné - sans que cela soit exclusif - deux points grammaticaux qui fonctionnent comme «moteurs de cette polyphonie ${ }^{6} »$ : le conditionnel et le discours rapporté. La question devient alors celle du rapport entre littéracie et grammaire. Nous allons d'abord développer ce point.

\section{Travailler la littéracie par la grammaire}

15 Nous sommes partis de textes à dominante argumentative extraits de journaux (Le Figaro, Libération, Le Monde) et avons sélectionné dans ce corpus les textes à utiliser (voir corpus de textes travaillés avec les apprenants, en annexe). Une argumentation n'est pas en soi polyphonique, mais dans un texte argumentatif le scripteur doit prendre en compte d'autres positions. Nous définirons la polyphonie comme l'intégration dans un texte donné d'éléments issus d'autres textes (niveau des traces) et donc comme l'insertion d'une parole autre (niveau des plans d'énonciation) ${ }^{7}$. 


\subsection{La prise en compte de la polyphonie à travers l'intertextualité}

16 L'intertextualité est le fait qu'un texte renvoie à d'autres textes préalables (le déjà-dit), ou produits dans le même temps (le dit-par-ailleurs), ou encore à venir (le dit-attendu). La polyphonie s'appuie ainsi sur l'intertextualité; mais la polyphonie n'est pas l'intertextualité et doit en être soigneusement distinguée. Alors que l'intertextualité est un phénomène transversal à la production et à la réception textuelle, la polyphonie est le jeu de traces, le dépôt de cette intertextualité dans les textes. Ce ne sont donc pas les phénomènes intertextuels que nous prendrons en compte mais ces traces repérables dans les textes.

\subsection{Le conditionnel}

$17 \mathrm{Au} \mathrm{XVIII}$ siècle, le conditionnel était considéré comme un mode particulier, nommé suppositif. Les travaux plus récents ont conduit à le penser soit comme un futur du passé, soit comme un mode non pas temporel mais épistémique (dédié à la croyance et au degré de prise en charge par le locuteur), une question centrale devenant « y a-t-il un ou deux "conditionnels"?» (Dendale \& Tasmowski, 2001), voire trois : temporel (futur du passé), épistémique, éventuel (soumis à hypothèse explicite ou implicite).

En tant que futur du passé (c'est-à-dire « futur vu depuis un passé »), le conditionnel ne dit rien sur la réalisation ou non du procès ${ }^{8}$. On peut aussi bien avoir (a) il espérait qu'il reviendrait et, heureusement, j'ai pu le revoir que (b) il espérait qu'il reviendrait, malheureusement le sort en a décidé autrement. Le doute sur la réalisation du procès n'est donc pas un corolaire de ce conditionnel sinon (a) contiendrait une contradiction. Il en est de même du conditionnel épistémique dit auparavant journalistique (voir Haillet, 2002 ; Kronning, 2012) qui n'exprime pas un doute mais « le refus de prendre en charge le contenu cognitif de son énoncé » (Kronning, 2012, p. 84). Ce faisant, il donne à voir la parole d'autrui ; il s'agit d'une assertion déléguée. Prenons un exemple (début d'un article travaillé avec les apprenants) :

(1)

La médiatisation de l'hostilité au projet de loi El Khomri était telle ces derniers jours que certains avaient fini par prendre leur désir pour la réalité. Le 9 mars, la gauche, la vraie, lycéens et étudiants en tête, descendrait ${ }^{9}$ en masse dans la rue. Et ce ne serait qu'un début... Certes, la météo n'était pas au rendez-vous, mais M.A. et ses amis frondeurs, P.M. et la CGT, le jeune W.M. et l'UNEF n'avaient pas, hier, de quoi pavoiser. La mobilisation ne fut pas à la hauteur de leurs espoirs. (Y. Thréard, " Jeunes mais pas dupes », Le Figaro, 10 mars 2016, p. 1.)

Si l'on arrête la lecture à " qu'un début », la connaissance des orientations politiques du Figaro fait que le lecteur est incité à considérer que descendrait et ne serait que sont des procès qui n'ont pas été validés ou qui ne le seront pas du point de vue du scripteur, ce qui montre que l'on peut être amené à prendre en compte des éléments intertextuels et ce que confirme la suite. Nous assistons ici à la mise en parallèle de deux positions : d'une part, celle de M.A., P.M., W.M. et de leurs partisans qui est suivie de conditionnels et, d'autre part, celle du scripteur. Le contexte droit de ces conditionnels confirme que ces deux positions divergent (alors que, dans l'énoncé (a), ci-dessus, les deux positions en présence convergeaient). 
Un passage discursif qui recourt au conditionnel permet donc de mettre en parallèle deux positions qui soit vont diverger, soit vont converger. C'est là le motif d'emploi du conditionnel. Mais cela a aussi un "effet» surtout lorsque les positions divergent : le lecteur peut être conduit à penser que l'information est douteuse, non fiable, etc. Il est important, dans le travail de lecture en langue étrangère, de ne pas confondre motif d'emploi et effet discursif dans le contexte : cela suppose d'apprendre à repérer les «fils discursifs » (un fil discursif est l'énoncé - au fil du texte - d'une position du scripteur ou d'un locuteur convoqué par le scripteur ; c'est la conséquence de la polyphonie $)^{10}$. Ces fils discursifs traduisent des positions différentes mais qui peuvent, ensuite, diverger ou converger.

21 La nécessaire consistance ou cohérence d'un discours suppose que les parallélismes de ce type situent les positions en présence les unes par rapport aux autres en les faisant converger (quand le scripteur prend à sa charge la position au conditionnel) ou diverger explicitement ou implicitement (quand le scripteur ne prend pas en charge la position au conditionnel).

\subsection{Discours rapporté et plans d'énonciation}

Nous nous limiterons dans cet article à un aspect du discours rapporté qui traverse discours direct et discours indirect: la modalité des verbes introducteurs (pour une présentation générale du discours rapporté, voir Rosier, 1999, 2008).

Prenons un exemple. Dans l'article "Juppé, "l'identité heureuse" en bandoulière " ( Le Monde du 15 septembre 2016, p. 8), que nous avons travaillé avec les apprenants, les propos rapportés d'Alain Juppé sont articulés aux propos du scripteur par les verbes introducteurs suivants (cette matrice, présentée ici horizontalement, a été donnée verticalement aux apprenants): [assumer-réaffirmer - lancer - ironiser - dénoncer regretter - déclarer - insister (deux fois) - souligner - assurer]. Chaque verbe introducteur, pris isolément, ne dit rien par lui-même, mais le cumul permet de dresser un portrait d'Alain Juppé vu par le scripteur: un personnage déterminé ${ }^{11}$ qui ne craint pas l'affrontement avec son adversaire. Pour chaque texte, on peut construire avec les apprenants les matrices des différents acteurs convoqués par l'article et mettre ainsi en évidence les différents rôles attribués.

24 Ajoutons que le conditionnel épistémique et le discours rapporté peuvent jouer chacun leur rôle en complémentarité ( $\mathrm{X}$ a proclamé qu'il serait candidat à l'élection pourtant cela n'a pas été le cas: ici, serait est à la fois un futur du passé par rapport à a proclamé et a une valeur épistémique par rapport à pourtant cela n'a pas étéle cas).

\section{Des apprenants de FLE face à la polyphonie textuelle}

25 Nous avons mené un travail empirique avec des apprenants de FLE afin de déterminer la façon dont ils prenaient conscience ou non de la polyphonie textuelle, mais aussi quels paramètres accentuer, dans le travail des textes, afin de faciliter leur accès à cette polyphonie. Nous pouvons maintenant analyser les données que nous avons recueillies lors des cours qui ont eu pour assise le lien dialectique entre analyses grammaticales et littéracie. 


\subsection{Présentation du dispositif et des données recueillies} choisissant des textes comportant des marques repérables de polyphonie (notamment conditionnel et discours rapporté). Cela nous a amenés à adopter un point de vue relevant non plus du discours mais de la littéracie (cf. section 2) et à travailler sur un thème unique donnant lieu à débat à l'époque du cours, à savoir la campagne présidentielle relatée et commentée dans trois quotidiens nationaux: Le Monde, Libération et Le Figaro (voir en annexe : corpus de textes travaillés avec les apprenants). Parce qu'ils traitent le même thème, ces textes s'éclairent les uns les autres tout en adoptant des points de vue différents du fait de l'orientation politique de chaque journal. Il y a constitution d'un intertexte commun aux apprenants, alimenté aussi par leur vécu en France, ce qui permet de traiter phrases elliptiques, titres comprenant une métaphore, rapports texte/dessin de presse, allusions à l'intertexte.

\subsection{Analyse des données : conditionnel et discours rapporté selon les apprenants}

Dans les exercices d'élucidation du conditionnel, nous avons constaté que certains apprenants, au lieu d'analyser le texte, commençaient par passer en revue mentalement les valeurs du conditionnel qu'ils avaient apprises, ce qui pouvait conduire à des réponses erronées, voire fantaisistes. Nous reviendrons sur ce point lors de l'analyse des grammaires pédagogiques.

30

D'autre part, il s'avère que les apprenants sont moins familiarisés avec le conditionnel épistémique qu'avec le conditionnel d'hypothèse. Ils interprètent facilement : 
(2)

Si les jeunes étaient réellement informés des dangers des drogues, ils n'auraient même pas envie d'essayer. (Extrait d'un courrier des lecteurs paru dans Le Monde, 5 novembre 2012.)

31 Mais ils ont plus de difficultés avec les conditionnels du type de ceux de l'exemple (1) cidessus, qu'ils considèrent comme l'expression de l'incertitude de l'auteur ou comme une supposition (ou possibilité) et non comme le déroulement par le scripteur de ce que nous avons appelé «fil discursif » permettant de convoquer une position autre dans le texte. En témoignent des réponses comme :

(3) Elles [les phrases] sont conditionnels ${ }^{13}$, c'est pour dire quelques choses qui ne sont pas certaines [incertitude].

(4)

L'auteur n'est pas sûr [incertitude], il a supposé la situation [supposition].

Cette difficulté d'explicitation de l'emploi du conditionnel a une implication dans la compréhension générale du texte. En effet, l'analyse de l'ensemble des réponses au questionnaire montre que les apprenants qui ne perçoivent pas la non-convergence des propos au conditionnel avec la position du journaliste ne comprennent pas le caractère polémique de ce premier paragraphe et se méprennent donc sur la visée argumentative de l'ensemble du texte.

Nous avons évoqué à la section 3 l'éventuelle complémentarité entre conditionnel et discours rapporté. Un exemple en est fourni par l'analyse de :

(5)

Une manière de se démarquer [...]. " C'était mieux avant », a-t-il [A. Juppé] ironisé, en dénonçant le «fatalisme» existant «chez certaines élites politiques ou intellectuelles ». " À les entendre, la France serait condamnée à la médiocrité, au déclin et à la peur ", a-t-il regretté. (A. Lemarié, "Juppé, "l'identité heureuse" en bandoulière ", Le Monde, 15 septembre 2016, p. 8.)

34 Nous pensions à priori que les nombreux guillemets présents dans cet énoncé favoriseraient le repérage de la polyphonie à laquelle participe le conditionnel, mais ce ne fut pas le cas pour la majorité. Un petit nombre d'apprenants (6 sur 16) répondent que le conditionnel permet de faire référence à un discours autre dont on peut ainsi se démarquer :

(6)

Le journaliste [...] montre que ce n'est pas lui qui fait cette conclusion. En fait, il prend la distance et fait la phrase plus objective.

Cependant, seulement l'un d'entre eux parvient à discerner qui prend ses distances (en l'occurrence A. Juppé) vis-à-vis de qui («certaines élites politiques »). La difficulté de ce passage réside en effet dans un emboitement des discours, puisque le journaliste cite un homme politique qui, lui, exprime son désaccord vis-à-vis des propos d'autres hommes politiques. Certains apprenants qui attribuent au journaliste la prise de distance peinent donc à percevoir toutes les facettes de la polyphonie orchestrée par ce dernier.

Concernant les matrices de verbes introducteurs de discours rapporté (c'est-à-dire les ensembles de verbes introducteurs utilisés dans un texte pour un locuteur donné, cf. section 3), matrices qui articulent le plan d'énonciation du scripteur avec les plans d'énonciation des locuteurs convoqués, on constate que, en fin de parcours d'apprentissage, seulement la moitié des apprenants réussit l'exercice. Parmi ceux qui échouent, la plupart fournissent des réponses inappropriées (cf. la réaction 
d'apprenant (7) ci-dessous); un apprenant fait un contresens par rapport à la signification du texte (8) tandis qu'un dernier apprenant omet de répondre.

(7)

Les caractéristiques mises en valeur d'Alain Juppé par ces termes sont franc, intelligent et modeste.

(8)

Le journaliste veut montrer les côtés faible et négatif de M. Juppé.

Tout porte à croire que les apprenants qui échouent ne parviennent pas à cumuler la signification de plusieurs verbes et ont tendance à interpréter mot par mot au lieu de «faire réseau ». D'ailleurs, un apprenant dit que A. Juppé est «insistant » parce que le verbe insister figure dans la matrice.

\section{Polyphonie et didactique}

Face aux difficultés rencontrées par les apprenants, il convient de s'interroger sur les outils disponibles et sur les approches didactiques qui permettront à ces derniers de dépasser leur appréhension trop limitée des textes. Les grammaires pédagogiques et certaines grammaires de référence font partie du panorama auquel est confronté un apprenant en FLE, c'est pourquoi nous les interrogerons. Ce travail nous permettra ensuite de mieux cadrer les préconisations que nous avons tirées de notre approche à la fois théorique et empirique.

\subsection{Grammaires pédagogiques et polyphonie}

Parce qu'elle tente de décrire de façon ordonnée des faits de langue à des fins d'efficacité pédagogique (Véronique, 2009), une grammaire pédagogique peut constituer un outil rassurant aussi bien pour l'enseignant que pour l'apprenant de langue étrangère. Cet outil peut-il se révéler également éclairant en ce qui concerne les phénomènes relevant de la polyphonie? Pour le vérifier, nous avons analysé comment ces phénomènes sont présentés dans cinq grammaires pédagogiques destinées à des enseignants et des apprenants de FLE de niveau intermédiaire et/ou avancé (cf. le corpus donné avant la bibliographie ; nous reprendrons les titres par mots-clés). Ces grammaires pédagogiques ne traitant pas de la polyphonie textuelle (malgré une ébauche, cf. ci-dessous), nous distinguerons leur approche du conditionnel et celle du discours rapporté. Nous avons aussi consulté une grammaire de référence : Riegel, Pellat et Rioul (1994-2016).

En confrontant les différentes valeurs attribuées au conditionnel dans les grammaires pédagogiques, on note tout d'abord qu'une seule fait l'unanimité : la valeur d'hypothèse/ éventualité. On trouve ensuite, d'une part, le futur du passé (considéré surtout comme relevant de la concordance des temps) et, d'autre part, le conditionnel de politesse (modalisation d'une demande) dans quatre grammaires sur cinq. En revanche, le conditionnel épistémique - dit alors journalistique - n'est signalé que dans trois grammaires. Comment ce dernier conditionnel est-il décrit? Grammaire vivante (1987), Grammaire expliquée (2002), et Nouvelle grammaire (2004) expliquent que cette valeur du conditionnel permet au locuteur de présenter une information «avec prudence " ( Grammaire expliquée), voire "une certaine réserve» (Grammaire vivante) ou encore de signaler le caractère hypothétique de l'information donnée (Nouvelle grammaire), tout en soulignant sa fréquence d'emploi dans la presse. Il n'est à aucun endroit indiqué que le 
conditionnel peut signaler un discours autre non pris en charge par le locuteur/scripteur et les exemples relèvent d'ailleurs de l'information factuelle et non de l'argumentation (par exemple, Selon certaines sources, l'actrice se trouverait actuellement en Argentine, dans Grammaire expliquée, p.154). Enfin, à côté de ces valeurs du conditionnel, ces grammaires FLE relient ce temps verbal à des actes de langage comme exprimer un regret, un désir, formuler un conseil, une suggestion, un reproche, etc., ébauchant ainsi un lien entre marqueurs grammaticaux et usages discursifs. En revanche, la grammaire de référence (Riegel, Pellat \& Rioul, 1994-2016) se concentre sur les valeurs du conditionnel en les expliquant beaucoup plus et en précisant la notion modale du potentiel, ce qui est plus conforme à la tradition grammaticale.

41 En ce qui concerne le discours rapporté ${ }^{14}$, il est inégalement traité dans les grammaires pédagogiques puisque deux d'entre elles ne le mentionnent pas du tout. Les trois autres grammaires décrivent essentiellement les différentes modifications à effectuer pour «passer mécaniquement» du discours direct au discours indirect (pronoms, marqueurs temporels, temps verbaux) et soulignent la grande variété des verbes introduisant le discours indirect. Concernant ce dernier point, la Grammaire utile (1991) se distingue des deux autres en ce qu'elle donne également de nombreux exemples de verbes permettant non seulement de citer, mais aussi de reformuler un discours en exprimant la position du locuteur par rapport à une autre personne ou par rapport à ce qu'elle dit, ce qui constitue une première approche de la polyphonie.

Quant au discours indirect libre, il n'est présenté que dans deux grammaires qui, de plus, insistent sur sa spécificité littéraire ${ }^{15}$. En revanche, la grammaire de référence (Riegel, Pellat \& Rioul, 1994-2016) développe une analyse du discours indirect libre et signale par ailleurs que le discours rapporté ne se limite pas aux phénomènes qu'elle décrit.

Force est de constater que ces descriptions dans les grammaires pédagogiques permettront difficilement aux apprenants d'interpréter adéquatement des énoncés convoquant de multiples voix, comme ceux dont il a été question dans cet article. S'il est vrai que les grammaires FLE, du fait de leur finalité, se doivent de simplifier les faits de langue décrits, il nous semble cependant que lorsqu'elles s'adressent à des apprenants de niveau avancé, elles devraient mieux prendre en compte les phénomènes relevant de la polyphonie textuelle, notamment en soulignant les différentes nuances que peut exprimer le conditionnel épistémique (non prise en charge du discours autre allant jusqu'au désaccord) et en approfondissant d'autres aspects du discours rapporté (les verbes introducteurs; des tournures comme selon $X$, d'après $X$; etc.).

Cela dit, une véritable appréhension de la polyphonie ne saurait se passer d'une analyse appropriée des textes : quelle(s) approche(s) privilégier?

\subsection{Quelle(s) approche(s) des textes ?}

Tout d'abord, l'observation des comportements des apprenants a mis en lumière la nécessité de les aider à remplacer une lecture trop linéaire et fragmentée par une lecture établissant des liens entre différents éléments qui s'éclairent mutuellement et qui, une fois mis en réseau, permettent une interprétation adéquate du texte. Par exemple, l'élucidation du conditionnel présent dans l'article du Monde ("À les entendre, la France serait condamnée à la médiocrité ", cf. l'énoncé complet (5) ci-dessus) est facilitée si on le rapproche des autres marques de polyphonie que comprend cet énoncé : À les entendre qui annonce clairement la parole d'autrui, les nombreux guillemets, la postposition des 
verbes introducteurs du discours direct, la modalité exprimée par les verbes ironiser, dénoncer et regretter. D'autre part, le sens de ce passage est également éclairé par d'autres parties du texte, voire le texte dans son ensemble, et également l'intertexte (autres textes lus avec les apprenants sur le même sujet ainsi que leurs propres lectures). Il s'agit donc d'inciter les apprenants à opérer ces rapprochements, par exemple en leur proposant différents parcours de lecture à l'intérieur d'un texte (aborder un aspect particulier du texte qui se trouve à plusieurs endroits; puis un autre, etc.) ou même entre plusieurs textes traitant le même sujet (commencer à lire un paragraphe d'un premier texte, puis le confronter à un passage d'un deuxième texte, etc.).

D'autre part, pour percevoir la polyphonie textuelle, l'apprenant doit prendre conscience que la compréhension d'un texte nécessite de repérer les différentes couches qui le composent et de conceptualiser l'articulation entre ces différentes couches. Cela nécessite à la fois un travail au niveau du discours et un travail au niveau grammatical. Nous nous limiterons ici à ce second aspect. Mais nous devons préciser au préalable que, comme dans le cas de la perception, le premier mouvement est top-down (cf. section 2) : il faut partir du texte et du processus de sa compréhension.

Concernant donc la compréhension du texte, il est important d'attirer l'attention des apprenants sur un paradoxe : bien que « responsable de son texte », le scripteur assemble des significations qui sont hétérogènes (considérer le conditionnel comme indiquant le doute du scripteur va à l'encontre de cette prise de conscience). On peut, à partir de là, leur faire mener une enquête : quelles sont les positions en présence et comment sontelles marquées? Lorsque des hypothèses ont été élaborées collectivement, il convient de les stabiliser, tout en gardant à l'esprit qu'aucune analyse grammaticale n'est définitive. Cette stabilisation suppose des catégorisations (classer les phénomènes en plusieurs groupes), des descriptions (du type "utiliser telle marque permet de») et des préconisations (du type « quand on rencontre telle marque, se poser telles questions »).

\section{Conclusion}

On peut considérer la notion de littéracie sous différents aspects :

- son aspect sociologique, ce qu'apporte, dans une société, le développement du « savoir lireécrire » (cf. Goody \& Watt, 1962) ;

- son aspect éducatif, le fait de passer d'un processus en deux étapes différenciées (apprendre à lire puis apprendre à écrire) à une " entrée dans l'écrit ", dans sa globalité ;

- on aspect discursif, ce en quoi la notion de littéracie peut « dépasser » notre conception du « discours ", la position adoptée ici.

Ce faisant, nous avons montré que la notion de polyphonie est fondamentale pour la notion de littéracie : les textes s'échangent parce qu'ils s'entrelacent; un humain ne peut négliger ce qu'a dit un autre humain et doit donc lui faire une place.

Partant de la notion de "feuilletage énonciatif» partiellement dérivée de celle de polyphonie, nous avons abouti à la notion de "parcours de lecture». Nous voudrions souligner l'importance de cette dernière : elle reflète le caractère transphrastique des textes, caractère souvent remarqué par l'analyse de discours; fonctionnellement, un texte n'est pas une succession de phrases (il ne l'est que du point de vue formel ou structurel). Du point de vue de la signification construite, c'est-à-dire du point de vue 
fonctionnel, un texte est un réseau d'informations qui doivent être prélevées au sein de phrases contigües ou non afin que le lecteur reconstruise ladite signification.

51 Le fait de s'appuyer sur la notion de littéracie pour positionner le processus grammatical a eu pour conséquence de partir des textes et de leur intertextualité pour aller vers les marques grammaticales. Nous ne pouvons développer ce point, mais ce processus est l'une des manifestations possibles de ce qui a été appelé «conceptualisation grammaticale » qui ne fait pas de la grammaire un stock de marques (c'est-à-dire un ensemble de données statiques à disposition) mais un ensemble de marques dynamiques (c'est-à-dire « en puissance » - la grammaire mentale de l'apprenant) au service de la signification textuelle.

\section{BIBLIOGRAPHIE}

\section{Corpus de grammaires pédagogiques}

BÉRARD, Évelyne \& LAVENNE, Christian. (1991). Grammaire utile du français. Paris : Hatier-Didier.

CAllamand, Monique. (1987). Grammaire vivante du français. Paris : Larousse.

Delatour, Yvonne, Jennepin, Dominique, LÉon-Dufour, Maylis \& Teyssier, Brigitte. (2004). Nouvelle grammaire du français. Cours de civilisation française de la Sorbonne. Paris : Hachette-FLE. Nouvelle édition de : Grammaire du français, 1991.

MONNERIE, Annie. (1987). Le français au présent. Paris : Didier-Hatier.

Poisson-Quinton, Sylvie, Mimran, Reine \& MAheo-Le CoAdic, Michèle. (2002). Grammaire expliquée $d u$ français. Niveau intermédiaire. Paris : CLE International.

\section{Références}

AzZOPARDI, Sophie \& BRES, Jacques. (2011). Temps verbal et énonciation : le conditionnel et le futur en français : l'un est dialogique, l'autre pas (souvent). Cahiers de praxématique, 56, 53-76. BEACCO, Jean-Claude. (1989). Un rendez-vous manqué ? Théories du discours et grammaire en didactique du français langue étrangère. Dans S. Moirand, R. Porquier \& R. Vivès (dir.), ... Et la grammaire, Le français dans le monde (p. 138-146), numéro spécial.

BESSE, Henri \& PoRQUIER, Rémi. (1984). Grammaires et didactique des langues. Paris : Hatier-CREDIF. BOURGUIGNON, Christiane \& FoERSTER, Cordula (dir.). (1993). La grammaire à quoi ça sert ? Lidil, 9. BRes, Jacques, Haillet, Pierre Patrick, Mellet, Sylvie, NølKe, Henning \& Rosier, Laurence (dir.). (2005). Dialogisme et polyphonie : approches linguistiques. Bruxelles : De Boeck.

CHERVEL, André. (1977). ... Et il fallut apprendre à écrire à tous les petits Français. Histoire de la grammaire scolaire. Paris : Payot.

CHISS, Jean-Louis. (2003). Article : « Littératie ». Dans J.-P. Cuq (dir.), Dictionnaire de didactique du français langue étrangère et seconde (p. 157-158). Paris : CLE International. 
Combettes, Bernard, Fresson Jacques \& ToMAsSOne, Roberte. (1979-1980). De la phrase au texte, manuels de $4^{\mathrm{e}}$ et de $3^{\mathrm{e}}$ des collèges. Paris : Delagrave.

DENDALE, Patrick \& TASMOWSKI, Liliane (dir.). (2001). Le conditionnel en français. Université de Metz. Goody, Jack \& WATT, Ian. (1972 [1962]). The Consequences of Literacy. Dans P. P. Giglioli (dir.), Language and Social Context (p. 311-357). Harmondsworth, UK : Penguin Books.

HAILLET, Pierre Patrick. (2002). Le conditionnel français : une approche polyphonique. Paris : Ophrys.

KRONNING, Hans. (2012). Le conditionnel épistémique : propriétés et fonctions discursives. Langue française, 173, 83-97.

Lehmann, Denis, Moirand, Sophie, Mariet, François \& Catalan, Robert. (1979). Lire en français les sciences économiques et sociales. Paris : Didier-CREDIF.

LEHMANN, Denis (dir.). (1980). Lecture fonctionnelle de textes de spécialité. Paris : Didier-CREDIF.

OCDE. (2000). La littératie à l'ère de l'information (Rapport). Disponible en ligne sur <www.oecd.org/ fr/edu/innovation-education/39438013.pdf> (consulté le 15 septembre 2017).

Portine, Henri. (1998). Conceptualisation-automatisation et didactique de la grammaire. Dans G. Legrand (dir.), Pour l'enseignement de la grammaire (p. 117-130). Lille : CRDP.

PORTINE, Henri. (1999). Didactique de la grammaire : vers une nouvelle enkuklios paideia? Spirale, 23, 195-209.

Riegel, Martin, Pellat, Jean-Christophe \& Rioul, René. (2016 [1994]). Grammaire méthodique du français. Paris : PUF.

ROSIER, Laurence. (1999). Le discours rapporté : histoire, théories, pratiques. Bruxelles : De Boeck \& Larcier.

ROSIER, Laurence. (2008). Le discours rapporté en français. Paris : Ophrys.

SINCLAIR, John McHardy \& COULTHARD, Richard Malcolm. (1975). Towards an Analysis of Discourse: The English Used by Teachers and Pupils. Londres : Oxford University Press.

TISSET, Carole (dir.). (1997). Métadiscours et enseignement/apprentissage des langues (vol. 1 \& 2). Linx, 36 et 37.

TOMASSONE, Roberte. (1996). Pour enseigner la grammaire. Paris : Delagrave.

VÉRONIQUE, Daniel. (2009). L'acquisition de la grammaire du français langue étrangère. Paris : Didier.

\section{ANNEXES}

Corpus de textes (articles de presse) travaillés avec les apprenants.

\section{Printemps 2016}

«Jeunes mais pas dupes », éditorial par Yves Thréard, Le Figaro, jeudi 10 mars 2016.

La Une de Libération, « Loi travail. L'avertissement », jeudi 10 mars 2016.

La Une du Figaro, jeudi 10 mars 2016.

«La loi El Khomri : un rejet constant », par Noémie Rousseau, Libération, jeudi 24 mars 2016. 
"Le sénat valide l'expérimentation des "salles de shoot" ", site internet du Monde (< www.lemonde.fr/sante/article/2015/09/17/>), 17 septembre 2015.

"Salles de shoot, aveu d'impuissance », courrier des lecteurs par Rémy Gigos, Le Monde, dimanche 4-lundi 5 novembre 2012.

«Matignon contre les salles de consommation de drogue ", par Laurent Maisonnat, Le Figaro, jeudi 12 aout 2010.

« Drogues : vers des salles de consommation?", par Laetitia Clavreul, Le Monde pour Direct Matin, lundi 28 juin 2010.

«"Mademoiselle" est partie... », par Mickaël Fonton, Valeurs actuelles, $1^{\mathrm{er}}$ mars 2012, p. 28-29.

\section{Automne 2016}

« "Identité", "religion" et "culture" de nouveau en débat à droite », par Déborah Claude, AFP Doc, mardi 14 juin 2016.

" Sarkozy lance sa campagne à toute allure pour rattraper Juppé », par Alexandre Lemarié, Le Monde, 27 aout 2016, p. 8.

"Alain Juppé compte bien développer le thème de "l'identité heureuse" ", Libération, 7 septembre 2016, p. 11.

« Des ministres prennent le contrepied de Valls sur le burkini », par Sophie de Ravinel, Le Figaro, vendredi 26 aout 2016, p. 4.

«L'affiche de la finale est-elle vraiment connue d'avance? », contrepoint de Guillaume Tabard, Le Figaro, 23 septembre 2016, p. 6.

"Juppé, “l'identité heureuse" en bandoulière », par Alexandre Lemarié, Le Monde, jeudi 15 septembre 2016, p. 8.

\section{NOTES}

1. <www.coe.int/t/dg4/linguistic/Source/Framework_FR.pdf>.

2. La notion de polyphonie textuelle dénote différents aspects. Voir par exemple Bres et coll. (2005). Nous reviendrons plus loin sur cette notion.

3. Du point de vue didactique, la grammaire est soit ancillaire (elle favorise l'activité discursive chez l'apprenant) soit autonome (la grammaire pour la grammaire). Pour nous, elle doit être ancillaire comme cela apparaitra plus loin.

4. D'après ce que je sais, il viendra [ou viendrait] demain n'engage pas de la même façon le locuteur.

5. Notre traduction. Cf. < www.unesco.org/new/en/education/themes/education-buildingblocks/literacy/>.

6. Le terme «moteur» est bien évidemment métaphorique. Il indique qu'il faut penser la polyphonie textuelle comme une dynamique, à laquelle contribuent le conditionnel et le discours rapporté.

7. Nous recourons volontairement à une définition minimale de la polyphonie. Notre propos n'est pas d'entrer dans les nombreux débats sur cette notion. On pourra, par exemple, se reporter à Bres et coll. (2005).

8. Voir à ce sujet Azzopardi et Brès (2011). 
9. C'est nous qui mettons les verbes au conditionnel en gras.

10. Il faudrait, ici, s'interroger sur les rapports entre ces « fils discursifs » et le discours indirect libre. Disons brièvement que si, dans un discours purement narratif, le conditionnel peut relever du discours indirect libre, dans un discours comportant de l'argumentation, même s'il y a du discours indirect libre, celui-ci est enchâssé dans les « fils discursifs » dont nous parlons ici.

11. Cela permet aussi un travail sur la relation de synonymie qui n'est pas une relation d'identité sémantique mais de convergence sémantique : déterminé peut être mis en rapport avec obstiné, tenace, têtu, volontaire.

12. Ce qui montre que la notion de texte n'est pas une évidence. Pour beaucoup d'apprenants, un texte énonce la position du scripteur et seulement sa position (sans l'idée d'éventuels débats internes). C'est l'une des raisons pour lesquelles la notion de concession est aussi une notion difficile à appréhender.

13. Nous avons conservé l'orthographe et les formulations des apprenants.

14. Notons que le terme "discours rapporté » n'apparait que dans la nouvelle édition de la grammaire des Cours de civilisation française de la Sorbonne intitulée Nouvelle grammaire (2004) : dans la première édition de 1991, il n'est question que de "discours direct " et "discours indirect ».

15. Ce n'est sans doute pas un hasard si la Nouvelle grammaire parle d'ailleurs de «style indirect libre » et non de « discours indirect libre».

\section{RÉSUMÉS}

Dans un premier temps, les auteurs dressent le panorama de l'évolution du rôle de la grammaire en didactique d'une langue, de la grammaire pour structurer ses énoncés à la grammaire pour le discours. Ils passent ensuite de la grammaire pour le discours à la grammaire pour la littéracie en travaillant sur la prise en compte de la polyphonie textuelle. Puis, ils décrivent deux marquages de cette polyphonie textuelle en français : le conditionnel et le discours rapporté. Ils présentent ensuite un travail empirique en classe de FLE à partir de textes de presse mettant en scène cette polyphonie. Ils étudient les réactions des apprenants et terminent sur des propositions didactiques.

The authors first provide a comprehensive overview of the evolution of the role of grammar in language learning, from grammar as structuration of utterances to grammar for discourse. Secondly they move on to grammar for "littéracie" by working on text polyphony. Then, they outline two markings of this polyphony in French language: French "conditionnel" and the reported speech. They continue by presenting an empirical work produced in "French as a Foreign Language" classes, drawing on press articles demonstrating the use of polyphony. They end with an analysis of how learners react and proposals for language learning. 
INDEX

Keywords : polyphony, grammar learning, literacy, written comprehension in a foreign language, grammar for learners

Mots-clés : polyphonie, didactique de la grammaire, littéracie, compréhension écrite en langue étrangère, grammaire pédagogique

\section{AUTEURS}

\section{MARIE-ODILE HIDDEN}

Université Bordeaux Montaigne, EA 4195 TELEM

\section{HENRI PORTINE}

Université Bordeaux Montaigne, EA 4195 TELEM 